\title{
Reducing the cesarean delivery rates for breech presentations: administration of spinal anesthesia facilitates manipulation to cephalic presentation, but is it cost saving?
}

\author{
Carolyn F Weiniger ${ }^{1,2^{*}}$, Paul S Spencer ${ }^{3}$, Yuval Weiss ${ }^{4}$, Gary Ginsberg ${ }^{5}$ and Yossef Ezra ${ }^{6}$
}

\begin{abstract}
Background: External cephalic version (ECV) is infrequently performed and $98 \%$ of breech presenting fetuses are delivered surgically. Neuraxial analgesia can increase the success rate of ECV significantly, potentially reducing cesarean delivery rates for breech presentation. The current study aims to determine whether the additional cost to the hospital of spinal anesthesia for ECV is offset by cost savings generated by reduced cesarean delivery.

Methods: In our tertiary hospital, three variables manpower, disposables, and fixed costs were calculated for ECV, ECV plus anesthetic doses of spinal block, vaginal delivery and cesarean delivery. Total procedure costs were compared for possible delivery pathways. Manpower data were obtained from management payroll, fixed costs by calculating cost/lifetime usage rate and disposables were micro-costed in 2008, expressed in 2013 NIS.
\end{abstract}

Results: Cesarean delivery is the most expensive option, 11670.54 NIS and vaginal delivery following successful ECV under spinal block costs 5497.2 NIS. ECV alone costs 960.21 NIS, ECV plus spinal anesthesia costs 1386.97 NIS. The highest individual cost items for vaginal, cesarean delivery and ECV were for manpower. Expensive fixed costs for cesarean delivery included operating room trays and postnatal hospitalization (minimum 3 days). ECV with spinal block is cheaper due to lower expected cesarean delivery rate and its lower associated costs.

Conclusions: The additional cost of the spinal anesthesia is offset by increased success rates for the ECV procedure resulting in reduction in the cesarean delivery rate.

Keywords: Breech, Cesarean delivery, Costs, Neuraxial blockade, Spinal

\section{Background}

Cesarean delivery is recommended by national guidelines for the breech presenting fetus (3-4\% of pregnancies) [1,2]. These same national guidelines recommend the availability of an external cephalic version (ECV) service; potentially enabling attempted vaginal delivery. Unfortunately, ECV is infrequently performed, hence $98 \%$ of breech presentations are delivered surgically [3,4]. Potential reasons for poor uptake of ECV include low ECV success rates, lack of

\footnotetext{
*Correspondence: carolynfweiniger@gmail.com

1 Department of Anesthesiology and Critical Care Medicine

Hadassah-Hebrew, University Medical Center, Ein Kerem, Jerusalem 91120, Israel

2Department of Anesthesia, Stanford School of Medicine, Stanford, CA, USA Full list of author information is available at the end of the article
}

physician referral and unwillingness of patients to undergo the procedure $[3,5,6]$.

Immediate hospital and health fund costs of cesarean delivery are higher than vaginal delivery $[7,8]$. A computerbased model of ECV trial versus scheduled cesarean delivery found that ECV is cost-effective above a success threshold of 32\% [7]. Addition of neuraxial blockade for ECV generates a hospital cost benefit if the ECV success rate is increased $11 \%$ above a baseline of $38 \%$ without neuraxial blockade [9]. However the study showing these data combined anesthetic (increased ECV success rate) and analgesic (little effect on ECV success) doses in their analysis; potentially muting the findings.

In ideal circumstances, vaginal delivery of a cephalic presentation is the safest route for both mother and neonate 
[10,11]. Uptake of ECV can be encouraged if success rates of ECV are maximally high; for example through use of neuraxial blockade [12-14].

The current study aims to determine whether the additional cost to the hospital of spinal anesthesia is offset by increased success rates for the ECV procedure; through decreased cesarean delivery rates.

\section{Methods}

The study was carried out in a tertiary hospital in the Labor and Delivery suite using financial data obtained from 2008 in New Israeli Shekels (NIS) and costs were adjusted to 2013 NIS using the consumer price index. The conversion factor used is 1.3336229. We present the costs to the hospital. Fixed equipment costs were calculated as a unit cost for the expected lifetime of the item. The fixed costs, for expenses such as hospital bed and multi-use equipment (blood pressure monitor, fetal heart rate monitor, and ultrasound) were calculated as a $20 \%$ overhead according to the accepted Israeli Ministry of Health method. Disposables include gloves, spinal needles, drugs, and sterile pack for performing anesthesia of surgery. Personnel include costs of all the staff (obstetricians, midwives and anesthesiologists) involved in each procedure: vaginal delivery, cesarean delivery and ECV with and without spinal anesthesia. Ethical approval was not required for this cost analysis study.

Current practice for management of breech presentation in our institution comprises performing ECV, or elective cesarean delivery. In the case of successful ECV vaginal delivery may be attempted.

We systematically searched Cochrane, Medline and Web of Science databases from 1990 to January 2013 using key words; "breech; external cephalic version; ECV; anesthesia; analgesia; spinal; epidural; neuraxial; version; fetal". We excluded manuscripts based upon title and abstract content. We manually searched the bibliography of relevant manuscripts. Using this search strategy we identified 6 randomized controlled trial publications using neuraxial blockade (comprising analgesia or anesthesia doses) for ECV and 3 cost analyses, and used these data to calculate our probability of ECV success with spinal anesthesia and delivery outcomes [7-9,15-20].

\section{Data analysis}

For each delivery management pathway the individual itemized costs for each major variable (fixed costs, disposables, and manpower) were entered as a separate item into an Excel worksheet (Microsoft Office 2000). Thus each delivery management pathway (vaginal delivery, cesarean delivery, spinal anesthesia) is presented as a unit total cost.

We pooled data from our two randomized controlled trials with an overall success rate of $76.1 \%$ for ECV using spinal anesthesia versus $44.8 \%$ ECV success rate without anesthesia - obtained from 134 enrolled patients (64 multiparas and 70 nulliparas) $[19,20]$. For our model, we used a vaginal delivery rate derived from the total rate of vaginal cephalic delivery following successful ECV in our pooled study population: 68 successful cephalic vaginal deliveries from 81 successful ECVs (84\%). Thus 16\% of successful ECV underwent cesarean delivery. This cesarean delivery rate was within the range reported among 206,909 deliveries between 2002-2008; the cesarean delivery rate for cephalic presentation intrapartum was $12.8 \%$, and for attempted vaginal delivery after labor induction was $21.1 \%$. Other studies reported a cesarean delivery rate after successful ECV of $16-26 \%$ [21,22].

Confidence intervals were calculated for the management pathways rates for cesarean and vaginal delivery using WinPepi version 11.15; using sample sizes from the derivation data $[19,20]$.

\section{Results}

Among 150,000 deliveries per year in Israel, up to $4 \%$ are breech presentation -6000 breech deliveries/year. In the case that $76 \%$ of ECV performed are successful using spinal anesthesia this would result in 4,560 potential vaginal deliveries. Of these, $16 \%$ may undergo cesarean, but 3830 (84\%) additional vaginal deliveries may occur.

The sum of the number of items and the costs per possible procedure performed are reported in Table 1. Cesarean delivery is the most expensive option, 11670.54 NIS, and vaginal delivery costs 4110.23 NIS. Successful ECV under spinal anesthesia with subsequent vaginal delivery costs 5497.2 NIS. Addition of spinal anesthesia for the ECV procedure costs 426.76 NIS. Using the costs presented in Table 1, maximum savings can be calculated: 8,321,820 NIS for spinal anesthesia for ECV of 6000 breech presentation cases, in addition to the delivery costs (3830 vaginal deliveries $=15,742,181$ NIS and 2170 cesarean deliveries $=25,325,072$ NIS) generating a total costs of 49, 389, 07 NIS. If all 6000 breech presentations were delivered by cesarean delivery without spinal anesthesia, the cost is 70, 023240 NIS, generating a maximum possible saving of 20,634, 167 NIS nationally in Israel per year.

The highest individual costs were for manpower for vaginal delivery, cesarean delivery and ECV. It was calculated that nursing care hours required were 14 per vaginal delivery, 4 per ECV procedure, 5.5 for cesarean delivery and physician hours would be 1.5, 2, and 3.5 for vaginal delivery, ECV and cesarean delivery, respectively. Expensive fixed costs for cesarean delivery include operating room trays and postnatal hospitalization (minimum 3 days).

Six randomized controlled trials (RCTs) report success rates for ECV, using neuraxial blockade compared to 
Table 1 Major variables reported per individual management option

\begin{tabular}{lccccc}
\hline $\begin{array}{l}\text { Total cost } \\
\text { (NIS) }\end{array}$ & $\begin{array}{c}\text { Personnel cost } \\
\text { (NIS) }\end{array}$ & $\begin{array}{c}\text { Disposable cost } \\
\text { (NIS) }\end{array}$ & $\begin{array}{c}\text { Fixed cost } \\
\text { (NIS) }\end{array}$ & No. items total per procedure & Procedure \\
\hline 11670.54 & 4076.89 & 1298.95 & 6294.70 & 77 & Cesarean delivery \\
4110.23 & 1824.40 & 250.72 & 2035.11 & 39 & Vaginal delivery \\
960.21 & 533.45 & 17.34 & 409.42 & 10 & ECV \\
1386.97 & 808.18 & 169.37 & 409.42 & 18 & ECV plus Spinal block \\
\hline
\end{tabular}

Key: Costs sourced in 2008 were adjusted to 2013 NIS price levels using the consumer price index. The conversion factor used is 1.3336229. (Central Bureau of Statistics, Price Statistics Monthly - September 2013. Jerusalem). Costs were sourced from hospital budget itemizations.

ECV = external cephalic version, NIS = New Israeli Shekel.

control, Table 2 [15-20]. The delivery rates in Figure 1 were retrieved from our previous studies for vaginal and cesarean delivery (combined multiparas and nulliparas) $[19,20]$. Previous RCTs using anesthesia for successful ECV reported a combined cesarean delivery rate of $16.3 \%[15,16,19,20]$. When analgesia, rather than anesthesia is considered for ECV, successful cases have a reported cesarean delivery rate of $14 \%[17,18]$. Table 3 is based upon the pathway rates presented in Figure 1. The costs associated with breech delivery are reduced as ECV success increases across a range of rates, and costs are additionally dependent on the cesarean delivery rate following successful ECV, Figure $2 \mathrm{a}$ and $\mathrm{b}$.

\section{Discussion}

In the current study we found that complications and quality of life considerations aside, adoption of policy of ECV with spinal anesthesia engenders hospital cost saving benefit through increased ECV success rates and subsequent decreased cesarean deliveries. The hospital cost of 426.76 NIS per anesthetic for ECV generates a cost saving of 1761.27 through decreased cesarean delivery - if the vaginal delivery rate following successful ECV is $84 \%$. For the 6,000 annual breech presenting births in Israel, this could save the hospital providers over 20 million NIS per year if ECV were performed under anesthesia for all breech presentation, and if ECV is maximally successful and cesarean delivery rates are minimal.
Several studies report that adopting a policy of ECV for the breech presenting fetus could result in a significant cost saving, in particular when neuraxial blockade is used [7-9]. Previously reported success rates of ECV, range from $30-70 \%[23,24]$. The current study considers ECV performed using spinal anesthesia, which significantly increases the ECV success rate to over 67\%. The success rates from ECV with neuraxial blockade vary considerably depending on whether an anesthetic or analgesic dose was used. Carvalho et al., demonstrated cost benefit for ECV using a combination of data from anesthesia and analgesia studies despite the latter being less effective at influencing ECV success rates [9]. Anesthetic rather than analgesia doses are more effective [25].

The ultimate aim of ECV is to reduce the cesarean delivery rates; breech cesarean delivery accounts for $25 \%$ of elective cases [26]. Following one cesarean delivery for breech presentation, subsequent cesarean delivery is expected in $90 \%$ of cases [27]. We calculated data based upon a $16 \%$ cesarean delivery rate following ECV success. Other studies report a wide range of cesarean delivery rates following successful ECV, from 16.4-50\% [21,28-31]. A lower overall cesarean delivery rate in a specific population undergoing vaginal delivery generates greater potential cost benefit using neuraxial block for ECV. Spinal anesthesia has a greater effect on the base case cost over the range of possible ECV success rates $(30-70 \%)$, if the prevailing cesarean delivery rate is $16 \%$.

Table 2 Vaginal delivery rates following ECV with neuraxial block for breech presentation (analgesia or anesthesia dose) in previously published randomized trials

\begin{tabular}{|c|c|c|c|c|c|}
\hline Authors & Analgesia/Anesthesia & $\begin{array}{l}\text { Effect size in } \\
\text { treatment group }\end{array}$ & $\begin{array}{l}\text { Effect size in } \\
\text { control group }\end{array}$ & $\begin{array}{l}\text { Vaginal cephalic delivery } \\
\text { in treatment group }\end{array}$ & $\begin{array}{l}\text { Vaginal cephalic delivery } \\
\text { in control group }\end{array}$ \\
\hline Dugoff [22] & Analgesia & $22 / 50$ (44\%) & $22 / 52(42 \%)$ & $16 / 50^{\ddagger}(32.0 \%)$ & $25 / 52^{\ddagger}(48.1 \%)$ \\
\hline Mancuso [20] & Anesthesia & $32 / 54(59 \%)$ & 18/54 (33.3\%) & $28 / 54^{a}(51.9 \%)$ & $13 / 54^{\mathrm{a}}(24.1 \%)$ \\
\hline Schorr [19] & Anesthesia & $24 / 35(69 \%)$ & $11 / 34(32.4 \%)$ & $23 / 35(65.7 \%)$ & $7 / 34(20.6 \%)$ \\
\hline Sullivan [21] & Analgesia & 22/47 (47\%) & 15/48 (31.3\%) & $17 / 47$ (36.2\%) & $12 / 48(25 \%)$ \\
\hline Weiniger [16] & Anesthesia & $24 / 36(67 \%)$ & $11 / 34(32.4 \%)$ & $16 / 36(44.4 \%)$ & $8 / 34$ (23.5\%) \\
\hline Weiniger [15] & Anesthesia & 27/31 (87\%) & 19/33 (57.6\%) & $25 / 31^{\mathrm{b}}(80 . \%)$ & $19 / 33^{b}(57.6 \%)$ \\
\hline
\end{tabular}

adata presented are vaginal cephalic delivery only.

Effect size = ECV success rate.

bexcludes vaginal breech delivery and repeat ECV.

${ }^{\ddagger}$ Following unsuccessful ECV five patients overall had spontaneous version to cephalic presentation with vaginal delivery. 


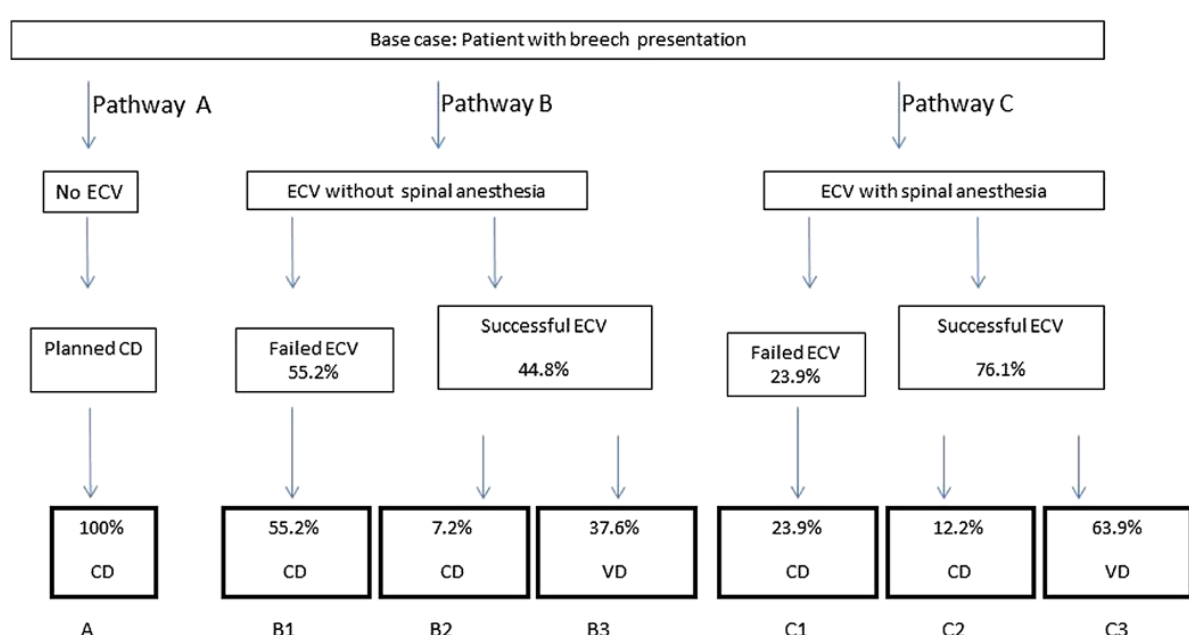

Figure 1 Potential delivery pathways for the base case patient with breech presenting fetus. The patient with breech presenting fetus can go directly to cesarean delivery, or undergo ECV, either with (Pathway B) or without (Pathway $\mathbf{C}$ ) spinal anesthesia. ECV success rates with and without spinal anesthesia were taken from our previous publications $[19,20]$. Pathway $\mathbf{A}$ (no ECV) shows $100 \%$ CD rate, despite the possibility of vaginal breech delivery or spontaneous conversion to cephalic presentation. For pathways B2/B3 and C2/C3, following successful ECV, a cesarean delivery rate of $16 \%$ is used, based upon calculated data from Table 3. Costs associated with each pathway are presented in Table 3 . VD =vaginal delivery, $C D=$ cesarean delivery.

When the cesarean delivery rate is $30 \%$, the cost differential between the ECV success ranges is less pronounced.

The current study micro-costed the hospital purchasing price of every item involved in the costs of performing each procedure (fixed, equipment, and personnel). Hence the actual costs of ECV, spinal block, normal delivery, and cesarean delivery, were calculated using the known itemized costs as has been done previously $[8,9]$. Other previous studies have used national department of health figures for calculating costs which are based on a national tariff or reimbursement fees, rather than actual itemized costs $[23,24]$. Costs differ greatly among countries. The cost of ECV in the USA is significantly higher than in the UK or in our current Israeli study $[7,8,15,32]$. The key factor in this cost variance is salary

Table 3 Costs for management pathways, derived from decision tree, Figure 1

\begin{tabular}{lllll}
\hline $\begin{array}{l}\text { Pathways for breech } \\
\text { management }\end{array}$ & $\begin{array}{l}\text { Management procedures } \\
\text { (ECV with/without spinal, } \\
\text { subsequent VD or CD) }\end{array}$ & $\begin{array}{l}\text { Unit cost } \\
\text { NIS }\end{array}$ & $\begin{array}{l}\text { CD/VD rates after ECV [19,20] Relative } \\
\text { probability (95\% CI) }\end{array}$ & $\begin{array}{l}\text { Relative cost NIS (CD rate } \\
\mathbf{1 6 \%} \text { after successful ECV) }\end{array}$ \\
\hline Pathway A & CD & 11670.54 & $100 \%$ & 11670.54 \\
No ECV & & Total pathway A & 6972.17 \\
Pathway B & & 12630.75 & $55.2 \%(0.40-0.64)$ & 909.41 \\
B1 & ECV (failure), CD & 12630.75 & $7.2 \%(0.03-0.16)$ & 1906.49 \\
B2 & ECV (success), CD & 5070.44 & $37.6 \%(0.26-0.49)$ & 9988.07 \\
B3 & ECV (success), VD & Total pathway B & 3120.74 \\
Pathway & & & 1593.02 \\
C1 & & 13057.51 & $23.9 \%(0.15-0.35)$ & 3512.71 \\
C2 & ECV + spinal, CD & 13057.51 & $12.2 \%(0.06-0.21)$ & 8226.47 \\
C3 & ECV + spinal, CD & 5497.2 & $63.9 \%(0.52-0.75)$ & Total pathway C \\
\hline
\end{tabular}

Key: Costs are derived from Table 1 unit costs and\% of each pathway occurring.

$C D=$ cesarean delivery, $V D=$ vaginal delivery, $E C V=$ external cephalic version, $\mathrm{Cl}=$ confidence interval.

Pathway $A=$ no ECV, Pathway $B=E C V$ without spinal anesthesia, Pathway $C=E C V$ with spinal anesthesia.

For $\mathrm{Cl}$ calculation, the denominator is 67 (Table 3) [19,20]. 


\section{a Sensitivity to ECV (no spinal) success rate}

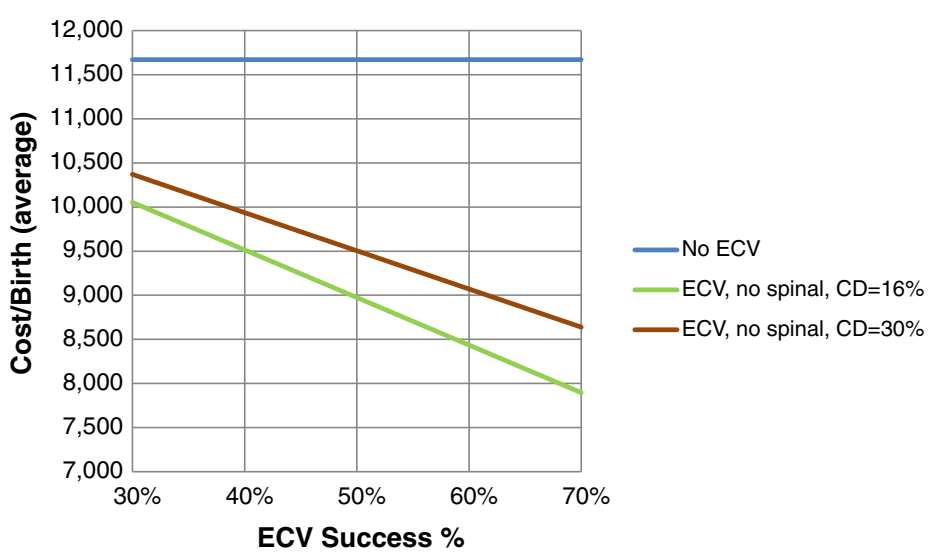

\section{b Sensitivity to ECV with spinal success rate}

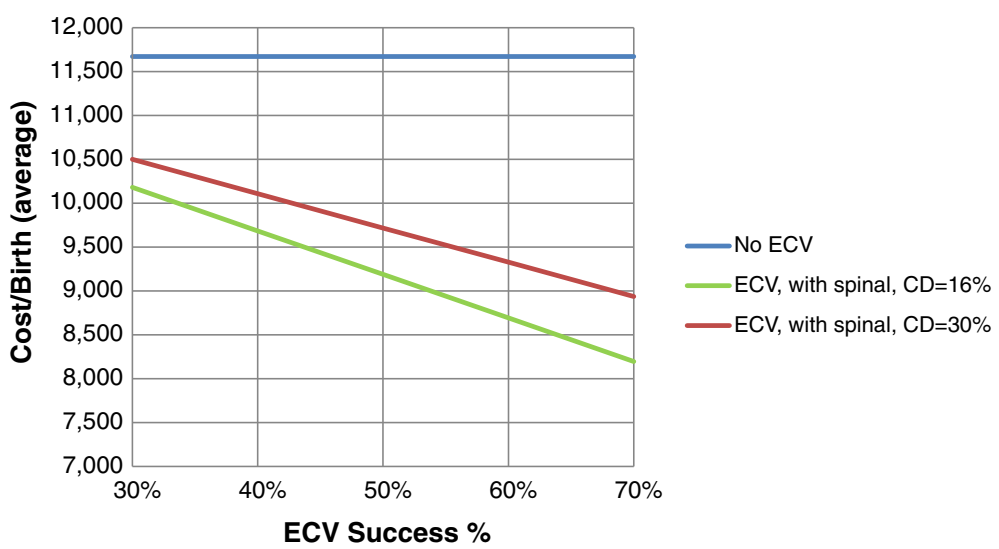

Figure 2 Reported ECV success rates range from 30-70\%; higher ECV success generate higher vaginal delivery rates - decreasing costs. Figure $2 \mathbf{a}$ shows ECV success rates ranging from $30-70 \%$ without spinal anesthesia and Figure $2 \mathbf{b}$ shows ECV success rates ranging from $30-70 \%$ with spinal anesthesia. Both show costs for a $16 \%$ and $30 \%$ cesarean delivery rate. If the prevailing cesarean delivery rate increases from $16 \%$ to $30 \%$, the cost savings from ECV success rates falls.

differences between the countries [33]. Recent data from the USA reported that ECV with neuraxial block costs 1221 \$ and without neuraxial block 1087 in 2010 [9]. This is almost four times the Israeli 2008 costs, however the same authors reported their ECV cost without neuraxial block in 2007 was only slightly lower, 1024\$. James et al. in a study from the UK, micro-costed each procedure involved in the ECV pathway, similar to the current study, and calculated ECV to cost approximately $284 \$$ (1136 NIS), only slightly higher than our local estimate [8]. Their study reported ECV but without neuraxial block, which may have significantly increased ECV success rates, thus providing a more remarkable cost saving. A cesarean delivery in the UK was also more expensive at $2930 \$$ compared with $2500 \$$ in Israel. The UK study did not factor in the cost of neuraxial block, and the average ECV success rate was only 50\%, less than that expected with use of spinal anesthesia in the current study. Since Ministry of Health imposes hospital costs in
Israel, ours may reflect those of other Israeli institutions. Although ECV is recommended by the American College of Obstetrics and Gynecologists (guidelines adopted in Israel), national ramifications of our study findings should consider that ECV practice may differ among institutions, as do cesarean rates.

A further cost consideration in the US is actual reimbursement to the hospital. The low USA reimbursement costs calculated on Relative Value Units (RVUs) for ECV (1.71) compared with cesarean delivery (16.33) may not encourage practice [34]. Personnel costs are considerably higher in the US compared with UK and Israel, albeit data for the UK are from 1997 [8,9]. Attempts to favor vaginal delivery through reimbursement have not curtailed the increase in cesarean delivery [4]. Hospital costs may be higher or lower than actual reimbursement costs, depending on how the hospital calculates their actual ECV costs.

Another important factor to consider is quality adjusted life years (QALYs) resulting from a particular 
delivery management plan. Factors affecting QALYs for cesarean delivery include maternal wound infection and duration of disability from surgical scar. Following normal vaginal delivery, relevant QALYS include immobility following perineal tears. The current study did not consider sufficient patients to calculate QALYs. However previous data suggest that normal vaginal delivery is less costly in terms of QALYs than cesarean delivery [7].

The data we present focus on hospital savings and ignore other potential costs benefits to the patient, family or health fund. The success rates of ECV under neuraxial block may differ between institutions, which would impact the savings found $[21,26,30]$. Any hospital wishing to calculate personal cost savings would need to make calculations based on their own ECV success and cesarean rates. Hospital stay, hence costs, are longer following cesarean and we did not calculate this benefit. Furthermore, cost of emergency cesarean delivery and the impact of spontaneous version were not calculated. Some women may refuse ECV, despite the possibility that ECV may be successful and allow them a vaginal delivery, or may have contraindications. Implementation of a formal decision management plan may increase ECV uptake [5]. Overheads (lighting, administration) are usually considered fixed costs and not itemized. Costs are calculated locally, and the overhead of $20 \%$ used in Israel may differ in other countries.

\section{Conclusions}

Performing ECV using spinal anesthesia results in large cost savings to the hospital overall, since the rate of cesarean delivery can be reduced. The potential savings in other countries, where unit procedure (specifically manpower) costs are higher, may be larger than those in Israel. Protocols for advising patients of the benefits of ECV, and the additional benefits of anesthesia should be implemented. Both the patient and the hospital stand to gain financially and otherwise from successful ECV with subsequent vaginal delivery.

\section{Competing interests}

The authors declare that they have no competing interests.

\section{Authors' contributions}

CFW and YE contributed to concept, design, analysis and preparation of the manuscript. PSS, YW and GG contributed to design, data analysis and content of the manuscript. All authors reviewed the final manuscript. All authors read and approved the final manuscript.

\section{Acknowledgements}

This study was supported (in part) by grant no. 6189 from the Chief Scientist Office of the Ministry of Health, Israel and a grant from the Hadassah-Hebrew University Medical Center Women's Health Fund.

\section{Author details}

'Department of Anesthesiology and Critical Care Medicine Hadassah-Hebrew, University Medical Center, Ein Kerem, Jerusalem 91120 Israel. ${ }^{2}$ Department of Anesthesia, Stanford School of Medicine, Stanford, CA, USA. ${ }^{3}$ Department of Electrical Engineering \& Computer Sciences, University of California at Berkley, California, USA. ${ }^{4}$ Hospital administration,
Hadassah-Hebrew University Medical Center, Ein Kerem, Israel. ${ }^{5}$ Department of Medical Technology Assessment, Ministry of Health, Jerusalem, Israel. ${ }^{6}$ Department of Obstetrics \& Gynecology, Hadassah-Hebrew University Medical Center, Ein Kerem, Jerusalem, Israel.

Received: 27 August 2013 Accepted: 9 January 2014 Published: 24 February 2014

\section{References}

1. Committee ACOG: on Obstetric Practice. ACOG Committee Opinion No. 340. Mode of term singleton breech delivery. Obstet Gynecol 2006, 108:235-237.

2. Green top guideline no. 20a: External cephalic version and reducing incidence of breech presentation. London: Royal College of Obstetricians and Gynaecologists; 2010. www.rcog.org.uk.

3. Cho LY, Lau WL, Lo TK, Tang HH, Leung WC: Predictors of successful outcomes after external cephalic version in singleton term breech pregnancies: a nine year historical cohort history. Hong Kong Med J 2012, 18:11-19.

4. Main EK, Morton CH, Melsop K, Hopkins D, Giuliani G, Gould JB: Cesarean deliveries, outcomes, and opportunities for change in California: toward a public agenda for maternity care safety and quality. California Medical Quality Care Collaborative White Paper 2011. www.cmacc.org/resources/ 2079/download. Retrieved November 202012.

5. Say R, Robson S, Thomson R: Helping pregnant women make better decisions: a systematic review of the benefits of patient decision aids in obstetrics. BMJ Open 2011, 21:1-e000261.

6. Vlemmix F, Kuitert M, Opmeer B, van der Post J, Mol BW, Kok M: Patient's willingness to opt for external cephalic version. J Psychom Obstet Gynecol 2013, 34:15-21.

7. Tan JM, Macario A, Carvalho B, Druzin ML, El-Sayed YY: Cost-effectiveness of external cephalic version for term breech presentation. BMC Pregnancy Childbirth 2010, 10:3.

8. James M, Hunt K, Burr R, Johanson R: A decision analytical cost analysis of offering ECV in a UK district general hospital. BMC Health Serv Res 2001, 1:6.

9. Carvalho B, Tan JM, Macario A, El-Sayed YY, Sultan P: A cost analysis of neuraxial anesthesia to facilitate external cephalic version for breech presentation. Anesthesia Analgesia 2013, 117:155-159.

10. Jackson N, Paterson-Brown S: Physical sequelae of caesarean section. Best Pract Res Clin Obstet Gynecol 2001, 15:49-61.

11. Kolas T, Saugstad OD, Daltveit AK, Nilsen ST, Oian P: Planned cesarean versus planned vaginal delivery at term: comparison of newborn infant outcomes. Am J Obstet Gynecol 2006, 195:1538-1543.

12. Sultan $P$, Carvalho B: Neuraxial blockade for external cephalic version: a systematic review. Int J Obstet Anesth 2011, 20:299-306.

13. Goetzinger KR, Harper LM, Tuuli MG, Macones GA, Colditz GA: Effect of regional anesthesia on the success rate of external cephalic version: a systematic review and meta-analysis. Obstet Gynecol 2011, 118:1137-1144.

14. Cluver C, Hofmeyr GJ, Gyte GM, Sinclair M: Interventions for helping to turn breech babies to head first presentation when using external cephalic version (Review) Cochrane Library. Cochrane Database Syst Rev 2012(1):CD000184.

15. Schorr SJ, Speights SE, Ross EL, Bofill JA, Rust OA, Norman PF, Morrison JC: $A$ randomized trial of epidural anesthesia to improve external cephalic version success. Am J Obstet Gynecol 1997, 177:1133-1137.

16. Mancuso KM, Yancey MK, Murphy JA, Markenson GR: Epidural analgesia for cephalic version: a randomized trial. Obstet Gynecol 2000, 95:648-651.

17. Sullivan JT, Grobman WA, Bauchat JR, Scavone BM, Grouper S, McCarthy RJ, Wong CA: A randomized controlled trial of the effect of combined spinal-epidural analgesia on the success of external cephalic version for breech presentation. Int J Obstet Anesth 2009, 18:328-334.

18. Dugoff $L$, Stamm CA, Jones OW 3rd, Mohling SI, Hawkins JL: The effect of spinal anesthesia on the success rate of external cephalic version: a randomized trial. Obstet Gynecol 1999, 93:345-349.

19. Weiniger CF, Ginosar Y, Elchalal U, Sela HY, Weissman C, Ezra Y: Randomized controlled trial of external cephalic version in term multiparae with or without spinal analgesia. Br J Anaesth 2010, 104:613-618.

20. Weiniger CF, Ginosar Y, Elchalal U, Sharon E, Nokrian M, Ezra Y: External cephalic version for breech presentation with or without spinal analgesia in nulliparous women at term: a randomized controlled trial. Obstet Gynecol 2007, 110:1343-1350. 
21. Gottvall T, Gintsman C: External cephalic version of non-cephalic presentation; is it worthwhile? Acta Obstet et Gynecol Scand 2011, 90:1443-1445.

22. El Toukhy T, Ramadan G, Maidman D, Hanna L, Waterstone M: Impact of parity on obstetric and neonatal outcome of external cephalic version. J Obstet Gynaecol 2007, 27:580-584.

23. Bolaji I, Alabi Isama L: Central neuraxial blockade-assisted external cephalic version in reducing caesarean section rate: systematic review and meta-analysis. Obstet Gynecol Int 2009, 2009:718981.

Epub 2009 Dec 23.

24. Gifford DS, Keeler E, Kahn KL: Reductions in cost and cesarean rate by routine use of external cephalic version: a decision analysis. Obstet Gynecol 1995, 85:930-936.

25. Lavoie A, Guay J: Anesthetic dose neuraxial blockade increases the success rate of external fetal version: a meta-analysis. Can J Anaesth 2010, 57:408-414.

26. Bragg F, Cromwell DA, Edozien LC, Gurol-Urganci I, Mahmood TA, Templeton A, van der Meulen JH: Variation in rates of caesarean section among English NHS trusts after accounting for maternal and clinical risks: cross sectional study. BMJ 2010, 341(c5056):1-8.

27. Menacker F, Hamilton BE: Recent trends in cesarean delivery in the United States. NCHS Data Brief (2010. No 35). http://www.cdc.gov/nchs/data/ databriefs/db35.pdf.

28. Bogner G, Xu F, Simbrunner C, Bacherer A, Reisenberger K: Single-institute experience, management, success rate and outcome after external cephalic version at term. Int J Gynecol Obstet 2012, 116:134-137.

29. Lau TK, Lo KW, Rogers M: Pregnancy outcome after successful external cephalic version for breech presentation at term. Am J Obstet Gynecol 1997, 176:218-223.

30. Preston R, Jee R: Anesthesia-facilitated external cephalic version: pennywise or pound-foolish? Can J Anesth 2013, 60:6-13.

31. Reinhard J, Sanger N, Hanker L, Reichenbach L, Yuan J, Herrmann E, Louwen $F$ : Delivery mode and neonatal outcome after a trial of external cephalic version (ECV): a prospective trial of vaginal breech versus cephalic delivery. Arch Gynecl Obstet 2013, 287:662-668.

32. Adams EK, Mauldin PD, Mauldin JG, Mayberry RM: Determining cost savings from attempted cephalic version in an inner city delivering population. Health Care Manag Sci 2000, 3:185-192.

33. Weissman C, Eidelman LA, Pizov R, Matot I, Klein N, Cohn R: The Israeli anesthesiology physician workforce. Isr Med Assoc J 2006, 8:255-260.

34. Changes to the CPT code set and medicare billing. http://www. obgmanagement.com/article_pages.asp?aid=10149 retrieved April 13th 2013.

doi:10.1186/2045-4015-3-5

Cite this article as: Weiniger et al: Reducing the cesarean delivery rates for breech presentations: administration of spinal anesthesia facilitates manipulation to cephalic presentation, but is it cost saving? Israel Journal of Health Policy Research 2014 3:5.

\section{Submit your next manuscript to BioMed Central and take full advantage of:}

- Convenient online submission

- Thorough peer review

- No space constraints or color figure charges

- Immediate publication on acceptance

- Inclusion in PubMed, CAS, Scopus and Google Scholar

- Research which is freely available for redistribution

Submit your manuscript at www.biomedcentral.com/submit
C Biomed Central 